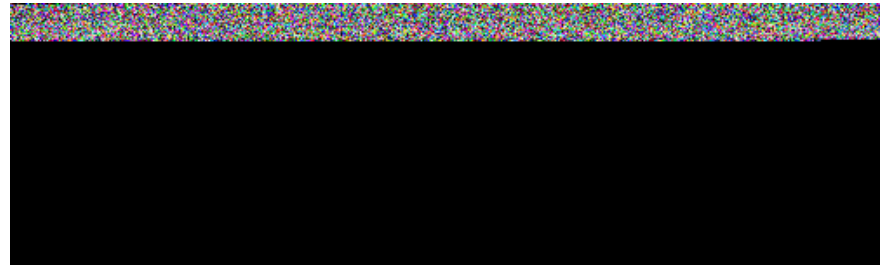

This information is current as of April 26, 2023.

\title{
Head and Neck Paragangliomas: Value of Contrast-Enhanced 3D MR
} Angiography

F. Neves, L. Huwart, G. Jourdan, D. Reizine, P. Herman, E. Vicaut and J.P. Guichard

AJNR Am J Neuroradiol 2008, 29 (5) 883-889

doi: https://doi.org/10.3174/ajnr.A0948

http://www.ajnr.org/content/29/5/883 


\section{ORIGINAL RESEARCH \\ Head and Neck Paragangliomas: Value of Contrast-Enhanced 3D MR Angiography}

F. Neves

L. Huwart

G. Jourdan

D. Reizine

P. Herman

E. Vicaut

J.P. Guichard
BACKGROUND AND PURPOSE: A rapid and accurate MR imaging technique would be beneficial to assess paragangliomas in the head and neck and to distinguish them from other lesions. The purpose of this study was to determine whether the combination of elliptic centric contrast-enhanced MR angiography (CE-MRA) and unenhanced and enhanced spin-echo imaging (conventional MR imaging) is more accurate than conventional MR imaging alone to assess paragangliomas in the head and neck.

MATERIALS AND METHODS: Three radiologists retrospectively and independently reviewed CE-MRA and conventional MR imaging in 27 patients with suspected paragangliomas. The overall image quality and the probability of paraganglioma were recorded. The results of each technique and their combination were analyzed for sensitivity and specificity. Receiver operating characteristic (ROC) analyses were performed by using histologic analysis, imaging, and/or clinical findings as the reference standard.

RESULTS: Forty-six lesions were found in 27 patients. In the assessment of paragangliomas, the combination of conventional MR imaging and CE-MRA was significantly superior to conventional MR imaging alone. Sensitivity and specificity respectively were the following: for CE-MRA, 100\% and 94\%; and for conventional MR imaging, $94 \%$ and $41 \%$. The specificity of CE-MRA was significantly higher than that of conventional MR imaging $(P=.004)$. There was good-to-excellent interobserver agreement for the paraganglioma probability with CE-MRA (nonweighted $\kappa, 0.67-0.77$ ), whereas there was fair-to-good interobserver agreement with conventional MR imaging (nonweighted $\kappa, 0.50-0.65$ ).

CONCLUSION: In combination with conventional MR imaging, CE-MRA yields an excellent diagnostic value for the assessment of head and neck paragangliomas; hence, the 2 techniques should be regarded as complementary.
$I^{n}$ $\mathrm{n}$ the head and neck, paragangliomas are slow-growing hypervascular lesions that are most commonly located in 4 main sites: the carotid bifurcation, the foramen jugulare, the middle ear cavity, and along the cervical portion of the vagus nerve. ${ }^{1}$ These lesions occur in both sporadic and hereditary forms ( $7 \%$ of cases). Multicentricity occurs in $10 \%$ of sporadic paragangliomas and in 30\%-40\% of familial paragangliomas.

Imaging techniques are used for suspected head and neck paragangliomas to confirm this diagnosis. ${ }^{2}$ Accurate assessment of tumor margins and invasion of adjacent structures are also essential for proper staging and therapy. ${ }^{3}$ For this task, MR imaging using unenhanced and enhanced fat-suppressed spin-echo (SE) sequences is widely accepted as the method of choice, ${ }^{4,5}$ in addition to high-resolution $\mathrm{CT},{ }^{6,7}$ in particular when the skull base is involved. Nonetheless, SE MR imaging has several limitations, particularly its sensitivity to artifacts. ${ }^{8}$ Moreover, the differentiation from other tumors and inflammatory lesions remains problematic. For example, the results of SE imaging are often equivocal when processes in the area of the jugular foramen are suspected. ${ }^{9,10}$ Somatostatin receptor scintigraphy (SRS) may be useful in this situation but is not always conclusive. Digital subtraction angiography (DSA) is the current imaging reference standard for assessing the vascular architecture of the tumor before embolic therapy, but this method is invasive with a complication rate of

Received July 5, 2007; accepted after revision December 1.

From the Departments of Neuroradiology (F.N., L.H., G.J., D.R., J.P.G.), Otorhinolaryngology (P.H.), and Statistics (E.V.), Groupe Hospitalier Lariboisière-Fernand-Widal AP-HP, Paris, France.

Please address correspondence to Jean-Pierre Guichard, MD, Service de Neuroradiologie, Hôpital Lariboisière, 2 rue Ambroise Paré, 75010 Paris, France; e-mail: jean-pierre. guichard@|rb.ap-hop-paris.fr

DOl 10.3174/ajnr.A0948
$0.5 \%-1 \%,{ }^{11}$ which is too high for a diagnostic examination. Consequently, some investigators have discussed the utility of more specific methods in MR imaging to differentiate paragangliomas from other tumors or vascular abnormalities (dynamic MR imaging, ${ }^{12}$ high-dose gadodiamide-injection dynamic MR imaging, ${ }^{13}$ or arterial and venous MR angiography $\left.[\mathrm{MRA}]^{9}\right)$.

To our knowledge, the elliptical centric contrast-enhanced MRA (CE-MRA) sequence has not been assessed for visualizing paragangliomas and differentiating them from other lesions. We postulated that CE-MRA has the potential to simplify the interpretation of conventional MR imaging because intense tumor blush on CE-MRA may be a sensitive and specific feature for paragangliomas.

The purpose of this study was to determine whether the combination of CE-MRA and conventional MR imaging is more accurate than conventional MR imaging alone to assess patients with suspected paragangliomas.

\section{Methods}

\section{Patients}

We reviewed retrospectively the records of 27 consecutive patients (19 women and 8 men; mean age, $45 \pm 14$ years; age range, $18-71$ years) referred between July 2002 and January 2006 because of known paragangliomas or in whom a paraganglioma was suspected on clinical (pulsatile tumor, tinnitus, familial paraganglioma) and/or previous imaging findings (ultrasound, CT, or MR imaging). They were assessed by a senior otorhinolaryngologist (with 20 years of experience in paragangliomas) for suggestion of paraganglioma, and all underwent the same SE MR imaging sequences and CE-MRA in our institution. The studied lesions included 34 paragangliomas (20 patients). Four of these 20 patients ( 5 paragangliomas) already had un- 
dergone surgery before MR imaging, and all had abnormalities suggestive of recurrent disease. Seven patients had multiple paragangliomas ( 1 of these patients had 5 localizations). Among these 7 patients, 5 had familial paragangliomas.

The other studied lesions (7 patients) were 3 schwannomas, 1 meningioma, 1 plasmocytoma, 1 venous malformation, and 1 dural arteriovenous fistula (DAVF).

\section{Reference Standard}

Histologic analysis was performed in 20 patients (25 lesions: 19 paragangliomas, 3 schwannomas, 1 meningioma, 1 plasmocytoma, and 1 lymphadenopathy) by an otorhinolaryngologic pathologist (with 15 years of experience). The interval between MR imaging and surgery ranged from 2 to 129 days (mean, $17 \pm 30$ days).

One DAVF was diagnosed with DSA.

In 6 patients, pathologic correlation was not necessary or not available: 5 patients had paragangliomas (elderly patients and/or bilateral localizations) and 1 patient had a venous malformation. In those 6 patients, both clinical and imaging findings were used as the reference standard:

- Clinical findings, CT, MR imaging, and SRS were all available for 3 patients and supported the diagnosis of paraganglioma.

- Two patients had a jugulotympanic paraganglioma, which had a typical aspect at otologic evaluation ( 1 of them had a hereditary paraganglioma). CT and MR imaging supported the diagnosis of paraganglioma.

- One patient had a venous malformation demonstrated on CT and MR imaging. It was treated successfully by percutaneous sclerotherapy.

Three lesions considered as paragangliomas were present in 1 patient and had a final diagnosis of reactive lymphadenopathies on clinical and imaging follow-up (repeated examinations of these lesions by MR imaging confirmed that they all disappeared). This patient with lymphadenopathies had a paraganglioma in another location, which was suggestive of multicentric disease.

\section{MR Imaging Technique}

MR images were available for all patients and were obtained with a 1.5T MR scanner (Signa; GE Healthcare, Milwaukee, Wis) with a neurovascular phased-array coil. For MR images (called conventional sequences), technical parameters were the following: axial unenhanced T1-weighted SE sequences (TR/TE, 500/11.7 ms; acquisition time [TA], 128 seconds); T2-weighted fat-suppressed fast SE sequences (TR/TE, 2840/98.5 ms; TA, 194 seconds); contrast-enhanced T1-weighted fat-suppressed SE sequences in the axial (TR/TE, 660/ $13.6 \mathrm{~ms}$; TA, 230 seconds) and coronal (TR/TE, 600/11.7 ms; TA, 207 seconds) planes, covering an area proximal from the carotid bifurcation to the skull base region to detect the most common localizations of paragangliomas (20 sections). All these SE sequences were performed with a matrix of $320 \times 224$, a FOV of $24 \times 18 \mathrm{~cm}$, and a section thickness of $4 \mathrm{~mm}$ with a $0.4-\mathrm{mm}$ intersection gap.

Moreover, a contrast-enhanced 3D fast time-of-flight spoiled gradient-recalled-echo sequence (CE-MRA) with elliptical $k$-space encoding was acquired in the coronal plane by using the following parameters: $\mathrm{TR} / \mathrm{TE}=6.2 / 1.5 \mathrm{~ms}$; flip angle, $30^{\circ}$; number of sections, 125 ; matrix size, $320 \times 224$, section thickness, $1.4 \mathrm{~mm}$; FOV, $30 \times$ $22.4 \mathrm{~cm}$; bandwidth, $31.25 \mathrm{kHz} ; 1$ excitation. Zero-filling interpolation was used. A 20-mL dose of gadoteric acid (Dotarem; Laboratoire Guerbet, Roissy, France) was injected into the antecubital vein with a flow rate of $2 \mathrm{~mL} / \mathrm{s}$, followed by a $25-\mathrm{mL}$ bolus of normal saline by using an automatic injector (Spectris; Medrad, Indianola, Pa). MR SmartPrep technique (GE Healthcare) or fluoroscopic triggering (Fluoro Trigger; GE Healthcare) was used to determine the time to maximal enhancement of the arteries. For these patients, the injection of gadolinium with a catheter was justified. Only a small additional dose of contrast medium is necessary for MRA. The acquisition time was 58 seconds, whereas that for the 4 SE sequences was 759 seconds (12 minutes 39 seconds). The preparation of the injector for the MRA sequence should be added to the duration of the examination.

\section{Image Analysis}

Conventional MR imaging images on the one hand and CE-MRA images on the other were independently reviewed by 3 observers blinded to the patient's history and to the final diagnosis. The reading order was randomized. One observer was a board-certified head and neck radiologist (with 15 years of experience) and the 2 others were junior radiologists (with 2 years of experience in radiology, including 6 months in a department of neuroradiology). For CE-MRA, images could be reformatted by both maximum intensity projection (MIP) and multiplanar reconstruction (MPR) techniques. The time available for interpretation was unrestricted. The 2 interpretation sessions were spread over 2 months, with at least a 1-month interval between them.

First, the readers were asked to rate the overall image quality on a 3 -point scale. On this scale, 3 corresponded to a good or excellent image quality; 2, to an acceptable image quality with confidence in diagnostic content; and 1, to an insufficient image quality. The presence of artifacts was assessed. The lesions were categorized into the following 5 locations: carotid, vagal, jugulotympanic, tympanic, or other. Readers were asked to give section numbers of the delineation of the mass to verify if they detected the same tumor. The greatest dimension was measured in the axial or coronal plane. Images were also analyzed for the presence or absence of flow voids. Finally, the observers were also asked to assess the probability of paraganglioma on a 3-point scale: 2 corresponded to probable; 1 , to uncertain; and zero, to improbable. If a lesion was not appreciated with one of the techniques but needed to be included in the analysis because it was seen with another technique, the unappreciated lesion was assigned to an "improbable" lesion in category zero. For conventional MR imaging, the diagnostic criteria were typical location, flow voids, typical displacement of internal and external carotids arteries (depending on the original site of the paraganglioma), and a "salt and pepper" aspect. For CE-MRA, the diagnostic criteria were location of the lesion and, above all, early and intense enhancement very similar to the arterial vascular enhancement. ${ }^{12}$ Consensus was always obtained in cases of discrepancy between readers.

\section{Statistical Analysis}

Statistical tests were calculated on the basis of the classifications of each observer and for the consensual results. For the diagnosis of paraganglioma, sensitivity, specificity, positive and negative predictive values (PPV and NPV), and accuracy of each combination of MR images (conventional and CE-MRA sequences) were calculated with equivocal cases considered positive. To compare the results obtained in the same patient with the 2 techniques, we used the McNemar test. A logistic regression model was fitted for the probability to detect a paraganglioma with the 2 different techniques. The prediction of each model was expressed by the area under the receiver operating characteristic (ROC) curve in logistic regression. 


\begin{tabular}{|c|c|c|c|c|}
\hline & Reader 1 & Reader 2 & Reader 3 & Consensus \\
\hline \multicolumn{5}{|c|}{ Sensitivity, \% } \\
\hline C MRI & 94.1 (80.32-99.28) & 94.1 (80.32-99.28) & $91.1(76.32-98.14)$ & 94 (80.32-99.28) \\
\hline CE-MRA & 97 (84.67-99.93) & 94.1 (80.32-99.28) & 97 (84.67-99.93) & $100(89.72-100)$ \\
\hline \multicolumn{5}{|c|}{ Specificity, \% } \\
\hline C MRI & 47 (22.98-72.19) & $35.2^{*}(14.21-61.67)$ & $52.9+(27.81-77.02)$ & $41 \ddagger(18.44-67.08)$ \\
\hline CE-MRA & $76.4(50.1-93.19)$ & $88.2^{*}(63.56-98.54)$ & $88.2+(63.56-98.54)$ & $94 \ddagger(71.31-99.85)$ \\
\hline \multicolumn{5}{|l|}{ PPV, \% } \\
\hline C MRI & 78 (62.39-89.44) & $74.4(58.83-86.48)$ & 79.5 (63.54-90.7) & $76.2(60.55-87.95)$ \\
\hline CE-MRA & 89.2 (74.58-96.97) & 94.1 (80.32-99.28) & 94.3 (80.84-99.3) & 97.1 (85.08-99.93) \\
\hline \multicolumn{5}{|l|}{ NPV, \% } \\
\hline C MRI & 80 (44.39-97.48) & $75(34.91-96.81)$ & 75 (42.81-94.51) & 77.8 (39.99-97.19) \\
\hline CE-MRA & $92.9(66.13-99.82)$ & $88.2(63.56-98.54)$ & 93.8 (69.77-99.84) & $100(79.41-100)$ \\
\hline \multicolumn{5}{|l|}{ Accuracy, \% } \\
\hline C MRI & $78.4(44.39-97.48)$ & $74.5(34.91-96.81)$ & $78.4(42.81-94.51)$ & 76.5 (39.99-97.19) \\
\hline CE-MRA & 90.2 (66.13-99.82) & $92.1(63.56-98.54)$ & 94.1 (69.77-99.84) & 98 (79.41-100) \\
\hline
\end{tabular}

Note:-C MRI indicates conventional MR imaging; in parenthesis, 95\% Cls; CE-MRA, contrast-enhanced MR angiography; PPV, positive predictive value; NPV, negative predictive value. ${ }^{*} \ddagger$ CE-MRA performed significantly superior compared with conventional MR Imaging.

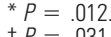

$\dagger P=.031$

$\ddagger P=.004$

To compare the performance of the conventional MR imaging alone with the performance of the combination of conventional MR imaging and CE-MRA, we conducted 2 logistic regressions for each reader. The area under the ROC curve $\left(\mathrm{A}_{z}\right)$ was compared by using the nonparametric statistical test described by DeLong et al. ${ }^{14}$

The $\kappa$ values were calculated for interobserver agreement. A $\kappa$ value $<0.40$ was considered to indicate poor agreement; $0.40-0.59$, fair agreement; 0.60-0.74, good agreement; and 0.75-1.00, excellent agreement.

Statistical analysis was performed by using SAS 9.13 (SAS Institute, Cary, NC). In all tests, the 2-sided significance level was fixed at 0.05 .

\section{Results}

A total of 46 lesions were identified in the 27 patients. Except contrary precision, consensual results of the 3 reviewers are given.

\section{Image Quality}

The global quality of conventional MR images was scored as good or excellent in $88.9 \%$, acceptable in $7.4 \%$, and insufficient in $3.7 \%$. The images for CE-MRA were considered as good or excellent in $96.3 \%(26 / 27)$ and acceptable in $3.7 \%$. Among the 27 studies, observers scored 1 MRA and $10 \mathrm{MR}$ imaging datasets as having susceptibility or motion artifacts.

\section{Paragangliomas Characteristics}

The studied lesions included 34 paragangliomas (20 patients). The paragangliomas' greatest dimension was $28 \pm 11 \mathrm{~mm}$ (range, $8-59 \mathrm{~mm}$ ). There were 16 glomus jugulares, 8 carotid body tumors, 8 vagal tumors, 1 retrothyroidian tumor, and 1 thoracic paraganglioma. Four paragangliomas were postoperative residues.

\section{Conventional MR Imaging}

For the diagnosis of paraganglioma, the results of sensitivity, specificity, PPV, NPV, and accuracy of each reader for both techniques are given in Table 1. MR imaging showed 10 falsepositive results ( 5 were classified as "probable" and the other 5 were classified as "uncertain"). These false-positive results were the following: 1 meningioma, 1 plasmocytoma, 4 reactive lymphadenopathies, 2 schwannomas, 1 DAVF, and 1 postoperative scar in a patient previously treated for paraganglioma. Uncertain findings (ie, rating of 1) were obtained with conventional MR imaging in 8 cases. Three paragangliomas were classified as "uncertain" on MR imaging. Two of these paragangliomas were postoperative residues, and 1 was malignant with cervical nodal metastases.

MR imaging detected flow voids in 30 of the 32 paragangliomas $(93.7 \%)$. The dimensions of the 2 paragangliomas that did not show flow voids on conventional MR imaging were 16 and $32 \mathrm{~mm}$. Conventional MR imaging showed flow voids in 5 other tumors (1 DAVF, 1 venous malformation, 1 schwannoma, 1 plasmocytoma, and 1 lymphadenopathy).

\section{CE-MRA}

All paragangliomas were detected with CE-MRA (sensitivity of $100 \%)$. There was a very early and strong contrast enhancement of paragangliomas (Figs 1 and 2) in comparison with the meningioma (Fig 3), the plasmocytoma, or the schwannomas (Fig 4). A minimal enhancement in CE-MRA was seen with the meningioma and the plasmocytoma. However, their enhancement was much less intense than that associated with paragangliomas. Only 1 lesion, which was not a paraganglioma, the DAVF, had an early and intense enhancement on CE-MRA. Uncertain findings (ie, rating of 1 ) were obtained with CE-MRA in this single case.

\section{Comparison of CE-MRA with Conventional MR Imaging}

In the assessment of paragangliomas, the $\mathrm{A}_{z}$ for the 3 reviewers for conventional MR imaging alone and the combination of conventional MR imaging and CE-MRA was 0.71 versus 0.89 $(P=.003), 0.65$ versus $0.93(P<.001)$, and 0.72 versus 0.93 $(P=.001)$, respectively. In the assessment of paragangliomas, the combination of the 2 techniques was significantly superior to conventional MR imaging alone.

For readers 2 and 3 and for the consensual results, the specificity of conventional MR imaging was significantly lower than that of CE-MRA (McNemar test, $P<.05)$. For reader 1 , the specificity for conventional MR imaging and MRA was 


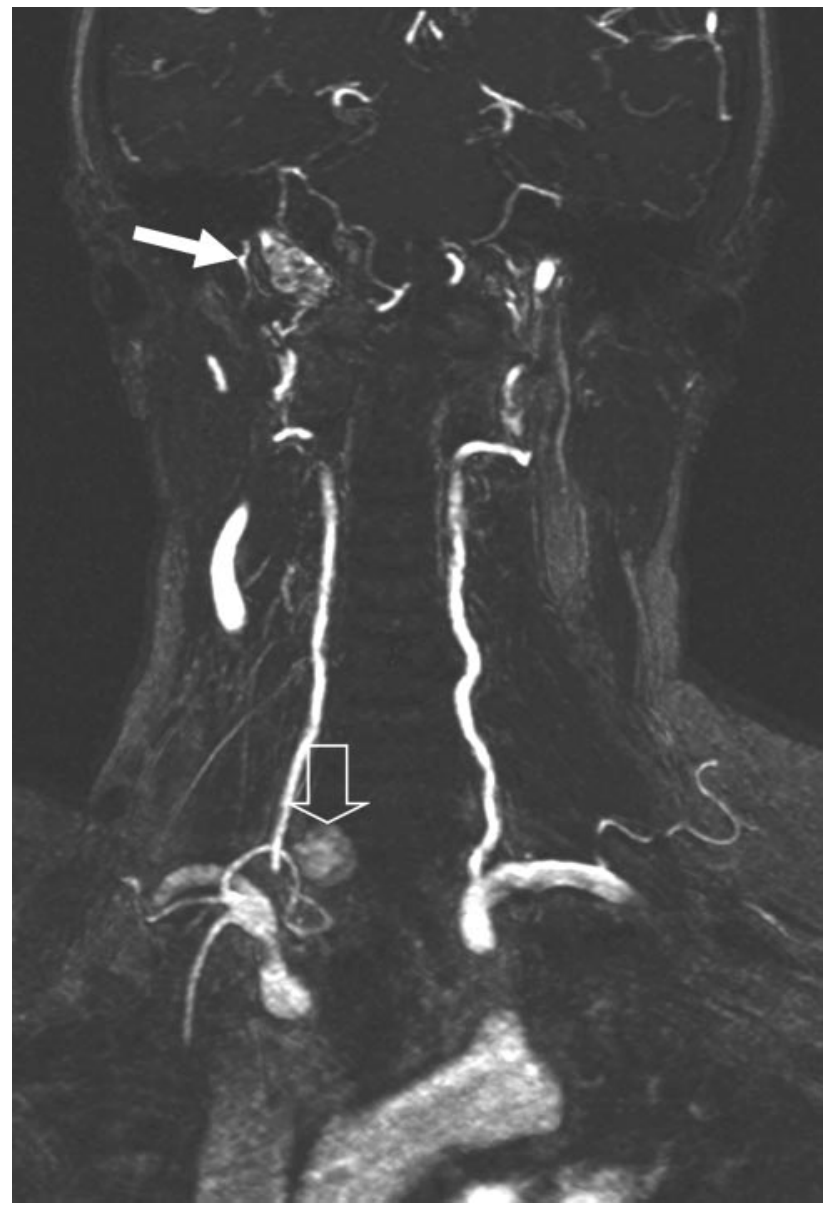

Fig 1. A 57-year-old woman with 2 paragangliomas: a residual vagal paraganglioma (solid arrow) and a retrothyroidian paraganglioma (open arrow). The second one was missed by DSA (because the feeding artery was not opacified) and by SE imaging (because it was situated out of the FOV, under the carotid bifurcation). They were depicted by CE-MRA and were both pathologically confirmed. CE-MRA coronal MIP image shows the 2 paragangliomas as intense tumor blush.

respectively $47 \%$ and $76.4 \%(P=.125)$. For each reader and for the consensual results, sensitivity, PPV, NPV, and accuracy with conventional MR imaging were lower than those obtained with CE-MRA (statistically not significant). An analysis performed with the equivocal cases (ie, rating of 1) considered negative instead of positive yielded similar results (better specificity but lower sensitivity for conventional MR imaging). The results concerning the uncertain findings for each reader were the following:

- There were 21 uncertain findings with conventional MR imaging. MRA allowed the correct diagnosis (rating of 2 for a paraganglioma or zero for another lesion) in 17 of these 21 cases $(81 \%)$.

- There were 15 uncertain findings with MRA. Conventional MR imaging allowed the correct diagnosis (rating of 2 for a paraganglioma or zero for another lesion) in 4 of these 15 cases $(27 \%)$.

The large field of view of CE-MRA sequence allowed the detection of 2 paragangliomas that were not visualized on conventional MR imaging. The first one was located in the superior mediastinum in a patient who had 5 paragangliomas. For this patient, all localizations (including the mediastinal one) showed an intense uptake with SRS. The second one was discovered by CE-MRA and was a retrothyroidian paraganglioma (Fig 1).

\section{Interobserver Agreement for Paraganglioma Probability}

For conventional MR imaging, the nonweighted $\kappa$ values were fair or good: 0.65 (confidence interval [CI], 0.40-0.90) between reviewers 1 and 2; 0.46 (CI, 0.15-0.78) between reviewers 1 and 3 ; and 0.50 (CI, 0.22-0.80) between reviewers 2 and 3 . The multirater $\kappa$ value was 0.54 (CI, 0.32-0.75).

For CE-MRA, the nonweighted $\kappa$ values were good or excellent: $0.71(\mathrm{CI}, 0.50-0.92)$ between reviewers 1 and $2 ; 0.67$ (CI, 0.45-0.89) between reviewers 1 and 3; and 0.77 (CI, 0.59$0.96)$ between reviewers 2 and 3 . The multirater $\kappa$ value was 0.72 (CI, 0.56-0.87).

For the $2 \mathrm{MR}$ imaging techniques, the difference between interobserver agreements was statistically not significant, but the $\kappa$ values for CE-MRA were higher than those for conventional MR imaging.

\section{Evaluation of Surgically Treated Paragangliomas}

Four patients ( 5 tumors) already had undergone embolization and surgery before MR imaging was performed. The tumors were suggestive of recurrent disease. In 1 patient, recurrence was confirmed by surgery. The other 3 patients did not undergo surgery again, but surgical data revealed a tumor residue in 3 patients and a complete treatment in 1 patient for a small carotid body tumor with no evidence of any local recurrence (clinical and imaging follow-up during 3 years). The probability of paraganglioma was assessed correctly in all cases with CE-MRA. In 3 cases, the probability of paraganglioma was assessed as "uncertain" with conventional MR imaging. Because of the small number of cases, statistical testing was not performed independently for them. The results of these 5 cases (4 patients) are given in Table 2.

\section{Discussion}

Previous studies have showed that paragangliomas have a typical temporal contrast enhancement. Their authors reported that this characteristic contrast enhancement can be useful in the diagnostic work-up of lesions that may mimic paraganglioma. ${ }^{12,13,15}$ All the techniques used in these studies were dynamic CE methods. Recently, improvements in gradients and sequences have made the use of elliptic centric CE-MRA possible to evaluate artery stenosis. ${ }^{16}$ For head and neck tumors, this sequence may offer anatomic and functional information about the vascular system. We postulated that intense tumor blush on CE-MRA could be a sensitive and specific feature for paragangliomas because these tumors are highly vascularized. ${ }^{17}$ Our study showed that with a sensitivity of $100 \%$ and a specificity of $94 \%$, CE-MRA was accurate to diagnose head and neck paragangliomas. Our results are in agreement with those studies concerning the typical rapid and intense enhancement of paragangliomas. However, the limitations of dynamic CE MR imaging are the small number of sections that can be acquired or the large section thickness. Contrary to dynamic CE MR imaging, CE-MRA can provide excellent spatial resolution and a large field of view, which are essential to explore paragangliomas. ${ }^{18}$ Previous studies ${ }^{18,19}$ have examined the sensitivity and specificity of unenhanced and CE 

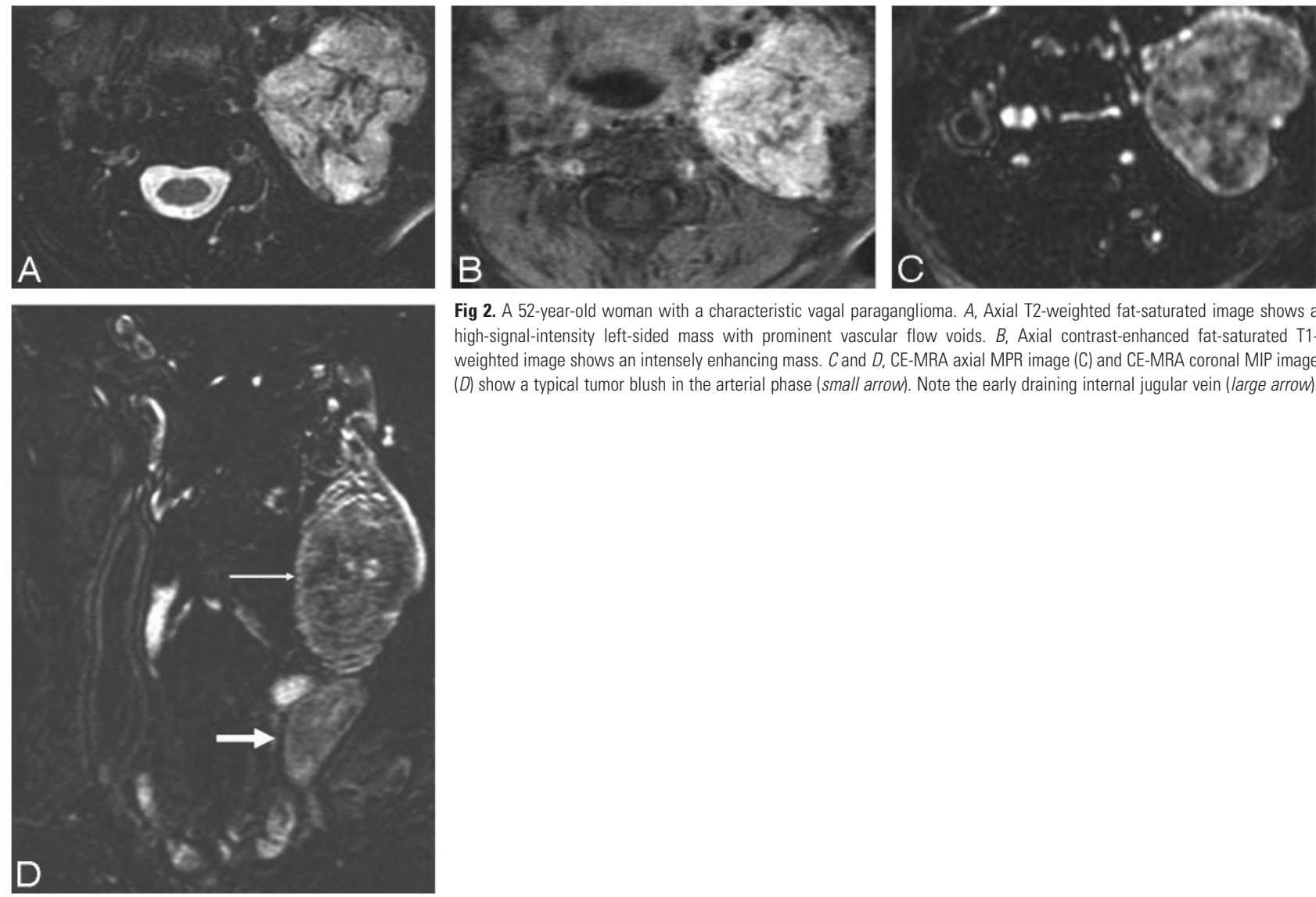

Fig 2. A 52-year-old woman with a characteristic vagal paraganglioma. $A$, Axial T2-weighted fat-saturated image shows a high-signal-intensity left-sided mass with prominent vascular flow voids. B, Axial contrast-enhanced fat-saturated T1weighted image shows an intensely enhancing mass. $C$ and $D$, CE-MRA axial MPR image (C) and CE-MRA coronal MIP image (D) show a typical tumor blush in the arterial phase (small arrow). Note the early draining internal jugular vein (large arrow)
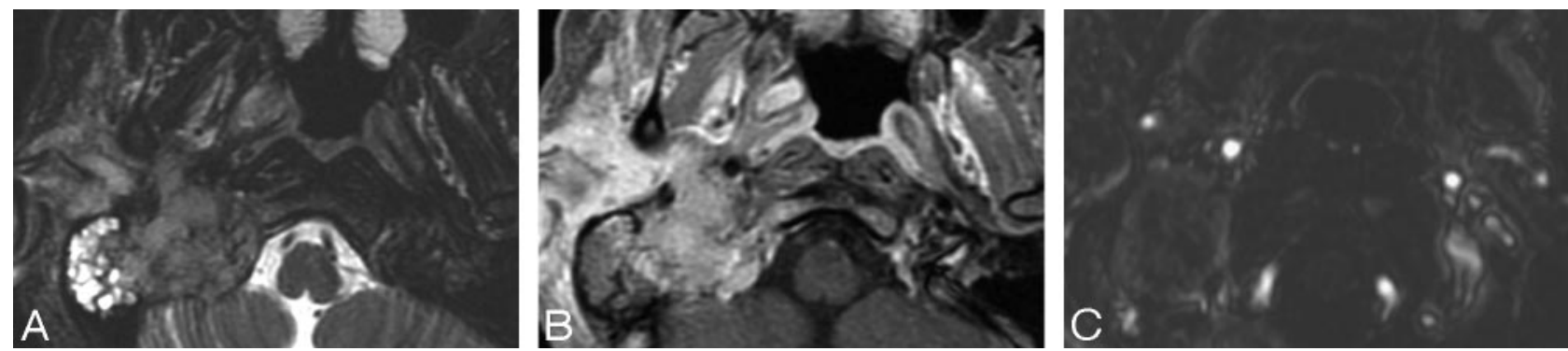

Fig 3. A 36-year-old woman with a primary jugular foramen meningioma. A, Axial T2-weighted fat-saturated image shows a mass centered in the right-sided jugular foramen with vascular flow voids mimicking a paraganglioma. $B$, Axial contrast-enhanced fat-saturated T1-weighted image shows strong enhancement of this mass. The tumor is difficult to differentiate from a paraganglioma on SE sequences. C, CE-MRA axial MPR image does not show the typical tumor blush in the arterial phase, which indicates that this tumor is probably not a paraganglioma.
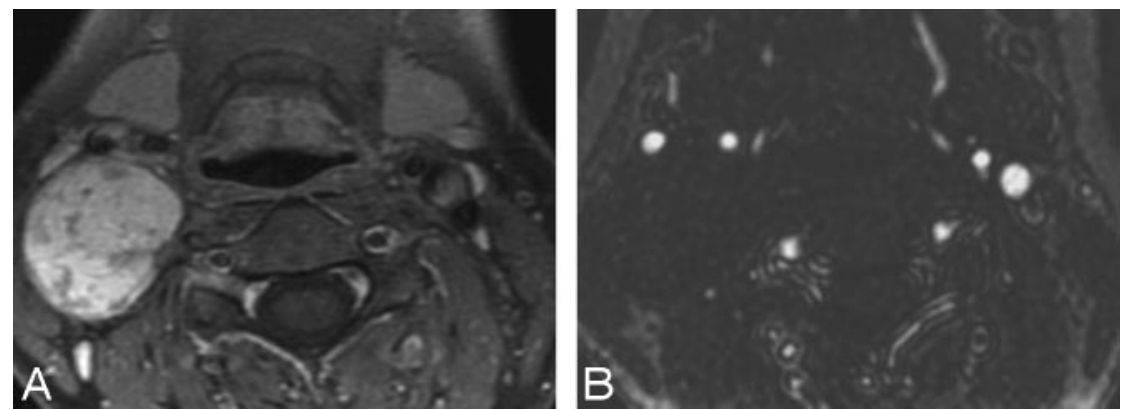

Fig 4. A 37-year-old woman with a cervical schwannoma. $A$, Axial contrast-enhanced fat-saturated T1-weighted image shows intensely enhancing well-circumscribed right-sided cervical mass. Differentiation with a paraganglioma is difficult. B, CE-MRA axial MPR image does not show the typical tumor blush in the arterial phase, which indicates that this tumor is probably not a paraganglioma. Note the anterior displacement of both the internal and external carotid arteries due to the schwannoma.

3D time-of-flight and have recommended these sequences to improve detection of paragangliomas. The major advantages of CE-MRA in comparison with 3D time-of-flight sequences are the rapid acquisition time and the large acquisition volume.

In our study, the typical angiographic appearance of a para- ganglioma (a hypervascular mass in a typical localization with intense tumor blush and early draining veins) described with DSA is also seen with CE-MRA. Paragangliomas showed this aspect on CE-MRA in all cases, allowing an accurate detection. The CE-MRA technique used in the present study had several advantages. 


\begin{tabular}{|c|c|c|c|c|c|c|}
\hline \multirow[b]{2}{*}{ Patient } & \multirow[b]{2}{*}{ Localization } & \multirow{2}{*}{$\begin{array}{l}\text { Interval Between } \\
\text { Surgery and MRI }\end{array}$} & \multirow{2}{*}{$\begin{array}{l}\text { Maximal Dimension } \\
(\mathrm{mm})\end{array}$} & \multicolumn{2}{|c|}{ Probability of PG } & \multirow{2}{*}{$\begin{array}{c}\text { Final Diagnosis } \\
\text { (reference standard) }\end{array}$} \\
\hline & & & & C MRI & CE-MRA & \\
\hline 1 & Vagal & 4 years & 20 & 1 & 2 & PG (surgery + pathology) \\
\hline 2 & Jugulotympanic & 9 months & 16 & 1 & 2 & $P G(S D)$ \\
\hline 3 & Vagal & 4 years & 22 & 2 & 2 & PG (SD) \\
\hline \multirow[t]{2}{*}{4} & Jugulotympanic & 6 months & 41 & 2 & 2 & PG (SD) \\
\hline & CBT & 6 months & 4 & 1 & 0 & Postoperative scar (follow-up) \\
\hline
\end{tabular}

Note:-CBT indicates carotid body tumor; C MRI, conventional MR imaging; PG, paraganglioma; SD, surgical data (known tumor residue); 2, probable; 1, uncertain; 0, improbable.

First, in the assessment of paragangliomas, the combination of conventional MR imaging and CE-MRA was significantly superior to conventional MR imaging alone. For 2 readers and for the consensual results, the specificity of CE-MRA was significantly higher than that of conventional MR imaging $(P<.05)$. CE-MRA caused a false-positive diagnosis in 1 case in comparison with 10 cases for MR imaging. This false-positive was a DAVF, which had an early and intense enhancement on CE-MRA. Nearly all the false-positive diagnoses of paraganglioma with conventional MR imaging were corrected by CE-MRA. The low specificity of conventional MR imaging may be explained by the high number of uncertain findings that where classified as positive for the statistical analysis. An analysis performed with the equivocal cases considered negative instead of positive yielded similar results for CE-MRA. Moreover, most of the lesions were seen with both techniques, but the confidence of the observers in determining the probability of paraganglioma was superior with CE-MRA. Indeed, uncertain findings (ie, rating of 1) were obtained with conventional MR imaging in 8 cases and in only 1 case with CE-MRA. Concerning the uncertain findings of each reader with conventional MR imaging, MRA allowed the correct diagnosis in $81 \%$ of the cases. Uncertain findings with MRA were corrected in only $27 \%$ of the cases by conventional MR imaging.

Second, CE-MRA is able to image from the aortic arch to the skull base, whereas most of MR imaging examinations for paragangliomas cover the temporal bone to the level of the carotid bifurcation. In our study, 2 lesions that were clearly detected by CE-MRA were outside the conventional MR imaging FOV.

Third, with a larger anatomic coverage, CE-MRA allows short acquisition times and yields excellent image quality in almost all examinations. Fat-suppression techniques are prone to magnetic susceptibility artifacts from air-tissue interfaces. ${ }^{8}$ They result in inhomogeneous fat suppression. Another limitation of conventional MR imaging includes its frequent exquisite sensitivity to motion artifacts because of a long acquisition time. 3D reconstructed images may be used with CE-MRA. The advantages of isotropic 3D imaging include the avoidance of partial volume artifacts and the potential to reformat images in any plane without loss of resolution.

Fourth, there was fair-to-good interobserver agreement in the paraganglioma probability with conventional MR imaging $(\kappa, 0.50-0.65)$, whereas there was good-to-excellent interobserver agreement in the paraganglioma probability with CEMRA $(\kappa, 0.67-0.77)$. The fact that we did not reach a significant difference between the 2 techniques is probably related to the small number of patients in this study.

Fifth, CE-MRA provided better differentiation of therapeutic changes from residual disease. The detection of residual or recurrent tumors after surgery and/or radiation therapy is difficult with SE imaging because of posttreatment changes, which may cause misinterpretation. However, evaluations with regard to postoperative residues were limited in this study because of the small number of cases (we experienced 4 residual paragangliomas). SRS has been recommended in this particular situation, ${ }^{20,21}$ but, to our knowledge, no results are available in large patient groups. CE-MRA, in addition to SRS, may be useful to confirm the complete tumor removal. Further studies are needed regarding the effectiveness of CE-MRA in this indication.

Paragangliomas typically show flow voids in SE imaging, ${ }^{22}$ but this feature is limited to lesions that are $>10 \mathrm{~mm} .{ }^{18}$ In this study, conventional MR imaging showed flow voids in 5 other tumors. Consequently, the signal-intensity voids within a tumor are not specific to paraganglioma, have shortcomings, and should be interpreted carefully, particularly in the skull base lesions.

However, CE-MRA sequences could sometimes be difficult to interpret because of fat surrounding the vessels. This fat has an intermediary signal intensity that can be difficult to differentiate from a very small ovoid blush.

CE-MRA is a noninvasive technique and has the advantage over DSA that it can display vascular structures in the axial plane without superimposition. Moreover, due to nonselective bolus application, CE-MRA visualizes all vessels within the selected volumes. It is useful when tumors fed by several arterial systems are only partially opacified in DSA or when they are in an unusual location. ${ }^{17}$ In 1 examination of a retrothyroidian paraganglioma, CE-MRA was the only examination to show this lesion, which was not visible on DSA, even in retrospect because the feeding arteries were not opacified during the selective angiography. The inclusion of CE-MRA in a standard imaging protocol could allow reducing substantially the practice of DSA to distinguish paraganglioma from an alternative diagnosis. Indeed, CE-MRA has a high specificity value for the diagnosis of paraganglioma, which is required to avoid an invasive angiographic procedure or a useless neck dissection. Thus, CE-MRA has the potential to replace DSA as the imaging reference standard.

SRS appears to be a reliable test to detect paragangliomas (sensitivity, $94 \%-97 \%$, specificity, $82 \%) .{ }^{21,23}$ It also appears useful in detecting synchronous tumors and in the screening of familial paragangliomas. ${ }^{20,24}$ Nonetheless, SRS has inconveniences: false-negative (small paragangliomas) and many false-positive results are known as in other neuroendocrine tumors, meningiomas, schwannomas, and small cell or breast carcinomas. ${ }^{23}$ In our study, 2 patients with schwannomas underwent SRS. The findings of the first were negative, the second had a focal uptake, but the activity was much less intense 
than that associated with known paragangliomas. SRS cannot differentiate paraganglioma from meningioma or plasmocytoma. Mutations involved in paragangliomas have now been characterized, but the penetrance of the mutation is variable. $^{25,26}$ SRS and MR imaging are used to screen patients with hereditary paraganglioma genes. ${ }^{27}$ Further studies are needed to study the accuracy of CE-MRA in this indication.

There are several potential sources of bias in our study. The first limitation was the lack of pathologic correlation in certain cases. Although the pathologic proof was not always present, there were strong arguments such as familial form or association with another characteristic localization of paraganglioma. Moreover, other tumors in the head and neck region such as hemangiopericytomas, endolymphatic sac tumors, and metastases from highly vascular tumors (eg, renal cell carcinoma) that may mimic paraganglioma were not present in this study. We also had a selection bias; the sensitivity and specificity that we obtained with MR imaging were seen in a preselected group in which the indication was to confirm or to rule out paragangliomas. Therefore, they do not reflect the true values for an unselected population. Last, this study had a relatively small number of patients. This can be explained by the fact that paragangliomas are rare tumors and CE-MRA is a recent technique.

We recommend adding CE-MRA to routine neck MR imaging when the clinical presentation evokes the diagnosis of paraganglioma (pulsatile mass, tinnitus, familial form) or when previous examinations showed arterial structures and a localization next to the jugular foramen, the hypotympanum, the carotid bifurcation, or the carotid space.

\section{Conclusion}

The typical appearance of a paraganglioma demonstrated with DSA is also seen with CE-MRA. CE-MRA is able to explore noninvasively and quickly a large field of view with an excellent image quality. Moreover, CE-MRA is a more specific technique than conventional MR imaging for assessment of head and neck paragangliomas and has the potential to provide the radiologists with added confidence in their interpretation. In the future, CE-MRA has the potential to replace DSA as the imaging reference standard for the diagnosis of paraganglioma. CE-MRA should be routinely used for suggested paraganglioma and for lesions of the skull base. It could be added to SE MR imaging for screening and follow-up of patients with hereditary paraganglioma genes.

\section{References}

1. Rao AB, Koeller KK, Adair CF. From the archives of the AFIP: paragangliomas of the head and neck-radiologic-pathologic correlation. Armed Forces Institute of Pathology. Radiographics 1999;19:1605-32

2. Lustrin ES, Palestro C, Vaheesan K. Radiographic evaluation and assessment of paragangliomas. Otolaryngol Clin North Am 2001;34:881-906, vi

3. Noujaim SE, Pattekar MA, Cacciarelli A, et al. Paraganglioma of the temporal bone: role of magnetic resonance imaging versus computed tomography. Top Magn Reson Imaging 2000;11:108-22

4. Ross MR, Schomer DF, Chappell P, et al. MR imaging of head and neck tumors: comparison of T1-weighted contrast-enhanced fat-suppressed images with conventional T2-weighted and fast spin-echo T2-weighted images. AJR Am J Roentgenol 1994;163:173-78

5. Mafee MF, Raofi B, Kumar A, et al. Glomus faciale, glomus jugulare, glomus tympanicum, glomus vagale, carotid body tumors, and simulating lesions: role of MR imaging. Radiol Clin North Am 2000;38:1059-76

6. Larson TC 3rd, Reese DF, McDonald TJ, et al. Glomus tympanicum chemodectomas: radiographic and clinical characteristics. Radiology 1987;163:801-06

7. Lo WW, Solti-Bohman LG, Lambert PR. High-resolution CT in the evaluation of glomus tumors of the temporal bone. Radiology 1984;150:737-42

8. Lewin JS, Curtin HD, Ross JS, et al. Fast spin-echo imaging of the neck: comparison with conventional spin-echo, utility of fat suppression, and evaluation of tissue contrast characteristics. AJNR Am J Neuroradiol 1994;15:1351-57

9. Vogl TJ, Juergens M, Balzer JO, et al. Glomus tumors of the skull base: combined use of MR angiography and spin-echo imaging. Radiology 1994;192:103-10

10. Caldemeyer KS, Mathews VP, Azzarelli B, et al. The jugular foramen: a review of anatomy, masses, and imaging characteristics. Radiographics 1997;17:1123-39

11. Heiserman JE, Dean BL, Hodak JA, et al. Neurologic complications of cerebra angiography. AJNR Am J Neuroradiol 1994;15:1401-07, discussion 1408-11

12. Arnold SM, Strecker R, Scheffler K, et al. Dynamic contrast enhancement of paragangliomas of the head and neck: evaluation with time-resolved 2D MR projection angiography. Eur Radiol 2003;13:1608-11

13. Vogl TJ, Mack MG, Juergens M, et al. Skull base tumors: gadodiamide injectionenhanced MR imaging - drop-out effect in the early enhancement pattern of paragangliomas versus different tumors. Radiology 1993;188:339-46

14. DeLong ER, DeLong DM, Clarke-Pearson DL. Comparing the areas under two or more correlated receiver operating characteristic curves: a nonparametric approach. Biometrics 1988;44:837-45

15. Nagele T, Petersen D, Klose U, et al. Dynamic contrast enhancement of intracranial tumors with snapshot-FLASH MR imaging. AJNR Am J Neuroradiol 1993;14:89-98

16. Wutke R, Lang W, Fellner C, et al. High-resolution, contrast-enhanced magnetic resonance angiography with elliptical centric $k$-space ordering of supraaortic arteries compared with selective $\mathrm{X}$-ray angiography. Stroke 2002;33:1522-29

17. Yoshikawa T, Aoki S, Hori M, et al. Time-resolved two-dimensional thick-slice magnetic resonance digital subtraction angiography in assessing brain tumors. Eur Radiol 2000;10:736-44

18. van den Berg R, Verbist BM, Mertens BJ, et al. Head and neck paragangliomas: improved tumor detection using contrast-enhanced 3D time-of-flight MR angiography as compared with fat-suppressed MR imaging techniques. AJNR Am J Neuroradiol 2004;25:863-70

19. van den Berg R. Imaging and management of head and neck paragangliomas. Eur Radiol 2005;15:1310-18

20. Duet M, Sauvaget E, Petelle B, et al. Clinical impact of somatostatin receptor scintigraphy in the management of paragangliomas of the head and neck. J Nucl Med 2003;44:1767-74

21. Kwekkeboom DJ, van Urk H, Pauw BK, et al. Octreotide scintigraphy for the detection of paragangliomas. J Nucl Med 1993;34:873-78

22. Olsen WL, Dillon WP, Kelly WM, et al. MR imaging of paragangliomas. AJR Am J Roentgenol 1987;148:201-04

23. Bustillo A, Telischi F, Weed D, et al. Octreotide scintigraphy in the head and neck. Laryngoscope. 2004;114:434-40

24. Whiteman ML, Serafini AN, Telischi FF, et al. ${ }^{111}$ In octreotide scintigraphy in the evaluation of head and neck lesions. AJNR Am J Neuroradiol 1997;18:1073-80

25. Neumann HP, Pawlu C, Peczkowska M, et al. Distinct clinical features of paraganglioma syndromes associated with SDHB and SDHD gene mutations. JAMA 2004;292:943-51. Erratum in: JAMA 2004;292:1686

26. Gimenez-Roqueplo AP, Favier J, Rustin P, et al. The R22X mutation of the SDHD gene in hereditary paraganglioma abolishes the enzymatic activity of complex II in the mitochondrial respiratory chain and activates the hypoxia pathway. Am J Hum Genet 2001;69:1186-97

27. Dundee P, Clancy B, Wagstaff S, et al. Paraganglioma: the role of genetic counselling and radiological screening. J Clin Neurosci 2005;12:464-66 